\title{
INSTRUMENTOS PARA AVALIAR A QUALIDADE E SEGURANÇA NO BLOCO OPERATÓRIO - REVISÃO INTEGRATIVA
}

\author{
José Augusto Pereira Gomes ${ }^{1}$, Maria Manuela Martins², Carla Sílvia Neves da Nova Fernandes ${ }^{3}$
}

\begin{abstract}
RESUMO: Este estudo teve como objectivo identificar os instrumentos existentes para avaliar a qualidade no bloco operatório. Realizou-se uma revisão integrativa da literatura no período entre 2010 e 2015 com o recurso a motores de busca específicos. Após a análise segundo os critérios de inclusão estabelecidos, foram seleccionados 13 artigos. A análise da evidência científica obtida permitiu organizar os resultados em três áreas temáticas: avaliação da estrutura, avaliação do processo e avaliação do resultado. Os instrumentos analisados são mais frequentes no âmbito do processo e associados à cultura de segurança do doente. Os resultados revelam a necessidade de maior desenvolvimento a este nível, nomeadamente através da construção e validação de instrumentos mais abrangentes, integrando as diferentes componentes da avaliação da qualidade.
\end{abstract}

DESCRITORES: Bloco operatório; Instrumentos; Qualidade nos cuidados.

\section{INSTRUMENTS TO EVALUATE QUALITY AND SAFETY IN THE SURGICAL CENTER - AN INTEGRATIVE REVIEW}

ABSTRACT: This study aimed to identify the instruments in existence for evaluating quality in the surgical center. An integrative review was undertaken of the literature in the period 2010 - 2015 through the use of specific search engines. Following analysis in accordance with the inclusion criteria established, 13 articles were selected. The analysis of the scientific evidence obtained made it possible to organize the results in three thematic areas: evaluation of the structure, evaluation of the process, and evaluation of the result. The instruments analyzed are more frequent in the ambit of the process and associated with the patient safety culture. The results reveal the need for further development at this level specifically through the construction and validation of instruments which are more broad-ranging, integrating the different components of the assessment of quality.

DESCRIPTORS: Surgical center; Instruments; Quality of health care.

\section{INSTRUMENTOS PARA EVALUAR LA CUALIDAD Y SEGURIDAD DEL BLOQUE OPERATORIO - REVISIÓN INTEGRATIVA}

RESUMEN: El estudio tuvo como finalidad identificar los instrumentos disponibles para evaluar la cualidad en el bloque operatorio. Se realizó una revisión integrativa de la literatura en el periodo entre 2010 y 2015 con el recurso de los motores de búsqueda específicos. Después del análisis de acuerdo con criterios de inclusión establecidos, fueron seleccionados 13 artículos. El análisis de la evidencia científica obtenida posibilitó organizar los resultados en tres áreas temáticas: evaluación de la estructura, evaluación del proceso y evaluación del resultado. Los instrumentos analizados son más frecuentes en el ámbito del proceso y asociados a la cultura de seguridad del enfermo. Los resultados apuntan la necesidad de más desarrollo en este nivel, específicamente por medio de la construcción y validación de instrumentos de acción más amplia, integrando los distintos componentes de evaluación de la cualidad.

DESCRIPTORES: Bloque operatorio; Instrumentos; Cualidad los cuidados.

${ }^{1}$ Enfermeiro. Doutorando em Ciências de Enfermagem no Instituto Ciências Biomédicas Abel Salazar. Enfermeiro especialista em enfermagem de Reabilitação. Porto, Portugal

${ }^{2}$ Doutora em Ciências de Enfermagem. Docente na Escola Superior de Enfermagem do Porto. Porto, Portugal

${ }^{3}$ Doutora em Ciências de Enfermagem. Docente na Escola Superior de Enfermagem Santa Maria. Porto, Portugal. 


\section{INTRODUÇÃO}

O bloco operatório é uma unidade orgânico-funcional constituída por um conjunto integrado de meios humanos, físicos e técnicos, destinados à prestação de tratamento cirúrgico ou realização de exames que requerem elevado nível de qualidade ${ }^{(1)}$. O bloco operatório é um dos contextos de trabalho mais complexos na área da saúde. Nestas unidades, as equipas de profissionais de saúde são altamente treinadas e qualificadas, exercendo a sua atividade em complementaridade e interagindo com tecnologia avançada, em situações de alto risco, com responsabilidade para responder às necessidades do doente cirúrgico(2).

Os serviços prestados em bloco operatório ao doente cirúrgico exigem uma adequada organização dos profissionais de saúde e de equipamentos, apoiados por procedimentos abrangentes e documentados, que reflitam uma prática de elevada qualidade ${ }^{(3)}$. As características dos ambientes perioperatórios colocam em risco de erro a equipa de saúde e em risco de ocorrência de eventos adversos, sendo que o maior número de erros ocorre do resultado dos cuidados e tratamentos prestados no bloco operatório(2).

O bloco operatório tem uma elevada prevalência de erros e acidentes, dos quais são exemplo a cirurgia errada, no doente errado, no órgão errado, no lado errado, retenção inadvertida de corpos estranhos e complicações cirúrgicas como a infeção e o tromboembolismo venoso, pelo que a segurança dos doentes no bloco operatório é um tema actual que recebeu recentemente grande atenção da Organização Mundial de Saúde (OMS), tendo-o tomado mesmo, como prioridade ${ }^{(4)}$.

Estima-se que os eventos adversos afetam 3-16\% de todos os doentes internados, sendo que mais de metade desses eventos são evitáveis. Apesar da evolução dos conhecimentos relacionados com a segurança cirúrgica, pelo menos metade dos eventos ocorrem durante o ato cirúrgico. Assumindo uma taxa de $3 \%$ de eventos adversos perioperatórios e uma taxa de $0,5 \%$ de mortalidade global, quase 7 milhões de doentes cirúrgicos terão complicações significativas em cada ano, 1 milhão dos quais morrerá durante ou imediatamente após a cirurgia ${ }^{(5)}$.

Neste sentido, é prioritária a implementação de medidas que garantam a qualidade destes cuidados ea segurança do próprio doente. Devido à complexidade da sua actividade e à sua natureza interdisciplinar, o bloco operatório constitui um campo vasto de atuação necessária para a implementação de medidas para garantir a qualidade total ${ }^{(6)}$, incluindo critérios de qualidade transversais a toda a organização e específicos do bloco operatório ${ }^{(2)}$. A introdução de modernas técnicas e modelos de gestão e o recurso a novos instrumentos e métodos de avaliação da qualidade dos serviços de saúde com o objectivo de imprimir eficiência, eficácia e rigor na gestão dos recursos, assim como corresponder às expectativas dos utentes, exige responsabilidades acrescidas nomeadamente aos enfermeiros.

Cientes destas responsabilidades e recorrendo a uma revisão integrativa da literatura, pretendemos identificar os instrumentos utilizados para avaliar a qualidade no bloco operatório que emergem da produção científica dos últimos anos, e com isso contribuir para a divulgação e incorporação dos mesmos, num instrumento único.

\section{MÉTODO}

O presente estudo pretende agrupar o conhecimento existente sobre os instrumentos para avaliar a qualidade no bloco operatório através de uma revisão integrativa. Tendo em consideração o conhecimento que se pretendia sintetizar, a revisão teve como ponto de partida a seguinte questão: "Que instrumentos são utilizados para avaliar a qualidade no bloco operatório?"

Após a identificação do tema e selecção da questão de pesquisa, foram estabelecidos os critérios de inclusão e exclusão de estudos. A pesquisa bibliográfica foi realizada entre novembro e dezembro de 2015. Foram seleccionados trabalhos publicados entre janeiro de 2010 e dezembro de 2015, utilizando como idioma o inglês, o espanhol e o português.

Para a revisão da literatura foi utilizada a base de dados referencial Scopus. O rastreio da literatura 
foi realizado tendo por base a utilização dos termos: Title-abs-key (Operating room OR operating theatre $O R$ surgery) and Title-abs-key (Instrument OR measure $O R$ tool) and Title-abs-key (Quality of care $O R$ Quality of health care) (Figura1), utilizando-se linguagem booleana para o cruzamento dos conjuntos em análise. A pesquisa foi limitada a título, palavras-chave ou descritores e resumo, com vista à blindagem do conjunto de documentos a rastrear. A escolha da base de dados Scopus fundamentou-se na incorporação das bases de dados bibliográficas EMBASE e MEDLINE, entre outras potencialmente relevantes ${ }^{(7)}$. Foram incluídos todos os artigos primários que utilizassem instrumentos para avaliar a qualidade e segurança no bloco operatório, tendo sido excluídos os artigos de revisão.

Após a leitura integral dos 36 artigos, a amostra final foi constituída por 13 artigos que atenderam aos critérios de inclusão. A análise do material foi realizada através de leitura crítica e qualitativa que permitiu identificar convergências, possibilitando o agrupamento por eixos temáticos. A análise crítica dos estudos incluídos é uma fase análoga à análise dos dados das pesquisas convencionais, esta fase obriga a uma abordagem organizada para ponderar as características de cada estudo ${ }^{(8)}$.

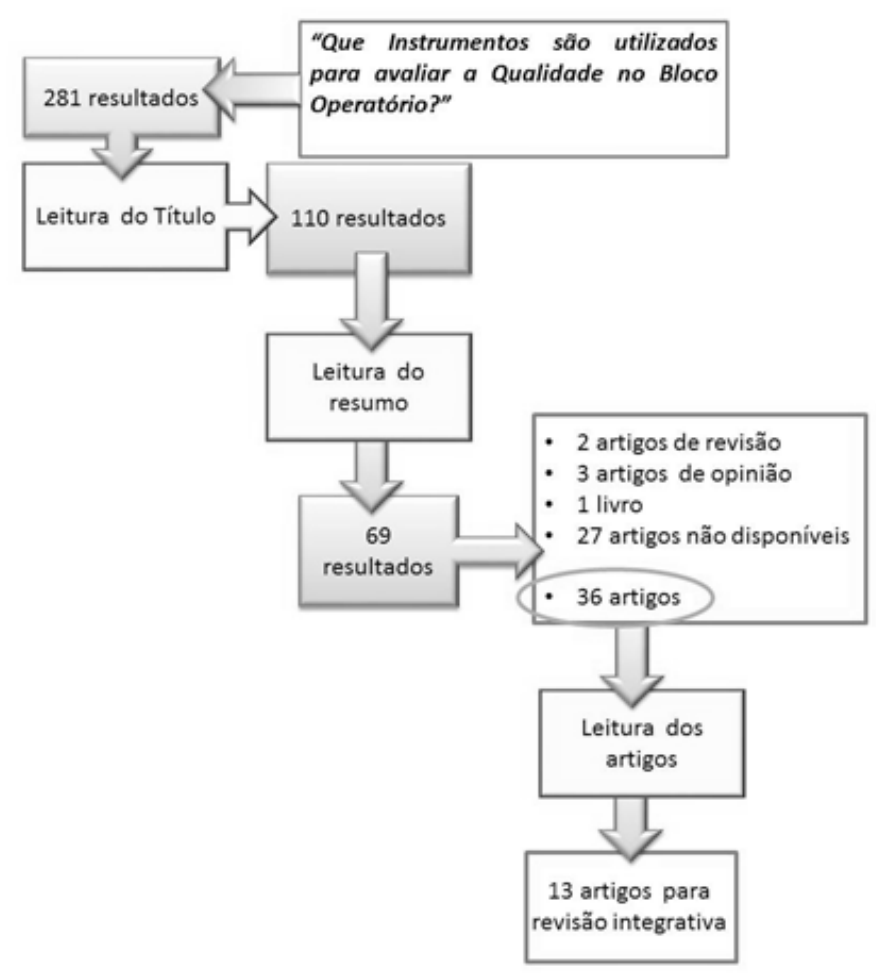

Figura 1 - Construção do protocolo de pesquisa. Porto-PT, 2016

\section{RESUlTADOS}

Os artigos foram categorizados quanto ao paradigma metodológico de estudo, um estudo era de natureza qualitativa e doze quantitativos, distribuindo-se pelos seguintes países: Reino Unido (5), Irlanda (1), Colômbia (1), Japão (1), Holanda (1), Espanha (1), Suécia (1), Turquia (1), EUA (1).

Em relação aos anos de publicação dos artigos, os artigos analisados apresentaram a distribuição apresentada na Figura 2, recorde-se que a pesquisa foi restringida ao espaço temporal entre 2010 e 2015.

Os resultados obtidos na pesquisa são visualizados na Tabela 1, na qual são identificados o título, os autores, o ano e a respetiva categorização.

Da análise dos estudos apresentados na Tabela 1, emergiram três eixos temáticos: a estrutura, o processo e os resultados. Esta tríade de aspectos essenciais para avaliação da qualidade tem por base o modelo de Donabedian. 


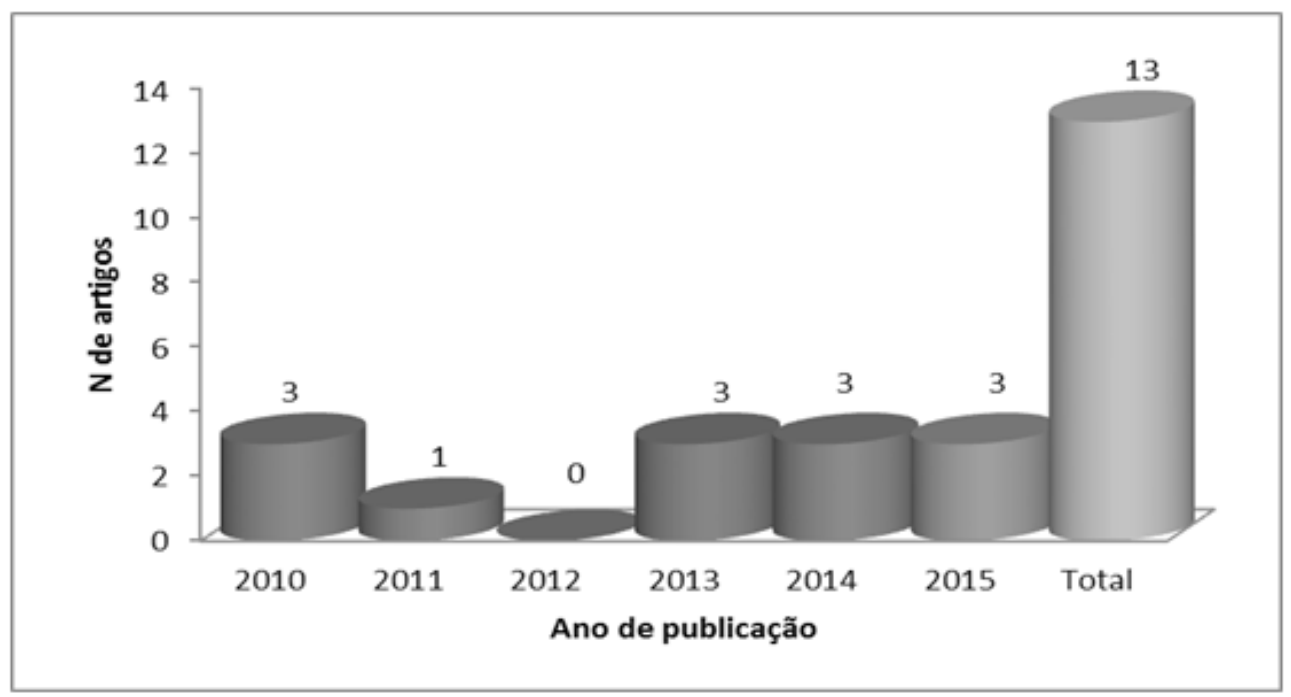

Figura 2 - Distribuição dos estudos, segundo o ano de publicação. Porto-PT, 2016

Tabela 1 - Artigos em análise. Porto-PT, 2016

\begin{tabular}{|c|c|c|c|c|}
\hline Categorias & Código & Autores & Título & Ano \\
\hline \multirow[t]{2}{*}{ Estrutura } & A1 & $\begin{array}{l}\text { Stepaniak; Vrijland; } \\
\text { Quelerij; Vries; Heij( }{ }^{(9)}\end{array}$ & $\begin{array}{l}\text { Working with a fixed operating room team on } \\
\text { consecutive similar cases and the effect on case } \\
\text { duration and turnover time }\end{array}$ & 2010 \\
\hline & $\mathrm{A} 2$ & Sedlack $^{(10)}$ & $\begin{array}{l}\text { Document The utilization of six sigma and statistical } \\
\text { process control techniques in surgical quality } \\
\text { improvement. }\end{array}$ & 2010 \\
\hline \multirow[t]{7}{*}{ Processo } & B1 & $\begin{array}{l}\text { Hill; Roberts; Alderson; } \\
\text { Gale }^{(11)}\end{array}$ & $\begin{array}{l}\text { Safety culture and the } 5 \text { steps to safer surgery: An } \\
\text { intervention study }\end{array}$ & 2015 \\
\hline & B2 & $\begin{array}{l}\text { Randmaa; Mårtensson; } \\
\text { Swenne; Engström }\end{array}$ & $\begin{array}{l}\text { SBAR improves communication and safety climate } \\
\text { and decreases incident reports due to communication } \\
\text { errors in an anaesthetic clinic: A prospective } \\
\text { intervention study }\end{array}$ & 2014 \\
\hline & B3 & $\begin{array}{l}\text { Kawano; Taniwaki; } \\
\text { Ogata; Sakamoto; } \\
\text { Yokoyama }^{(13)}\end{array}$ & $\begin{array}{l}\text { Improvement of teamwork and safety climate } \\
\text { following implementation of the WHO surgical safety } \\
\text { checklist at a university hospital in Japan }\end{array}$ & 2014 \\
\hline & B4 & $\begin{array}{l}\text { Arias; Barajas; Eslava- } \\
\text { Schmalbach; Hull; } \\
\text { Sevdalis }{ }^{(14)}\end{array}$ & $\begin{array}{l}\text { Translation, cultural adaptation and content re- } \\
\text { validation of the observational teamwork assessment } \\
\text { for surgery tool }\end{array}$ & 2014 \\
\hline & B5 & $\begin{array}{l}\text { Pickering; Robertson; } \\
\text { Griffin; Collins; } \\
\text { McCulloch }\end{array}$ & $\begin{array}{l}\text { Compliance and use of the World Health Organization } \\
\text { checklist in UK operating theatres }\end{array}$ & 2013 \\
\hline & B6 & $\begin{array}{l}\text { Papaspyros; Javangula; } \\
\text { Adluri, O'Regan }\end{array}$ & $\begin{array}{l}\text { Briefing and debriefing in the cardiac operating room. } \\
\text { Analysis of impact on theatre team attitude and patient } \\
\text { safety }\end{array}$ & 2010 \\
\hline & B7 & $\begin{array}{l}\text { Jothiraj; Howland-Harris; } \\
\text { Evley; Moppett }{ }^{(17)}\end{array}$ & $\begin{array}{l}\text { Distractions and the anaesthetist: A qualitative study } \\
\text { of context and direction of distraction }\end{array}$ & 2013 \\
\hline \multirow[t]{4}{*}{ Resultado } & C1 & $\begin{array}{l}\text { Sukha; Sykes; Schofield; } \\
\text { Houghton }{ }^{(18)}\end{array}$ & $\begin{array}{l}\text { Inadvertent returns to theatre within } 30 \text { days (IRT30) } \\
\text { of surgery: An educational tool to monitor surgical } \\
\text { complications and improve our performance as } \\
\text { surgeons }\end{array}$ & 2015 \\
\hline & C2 & $\begin{array}{l}\text { Bernalte-Marti; Orts- } \\
\text { Cortés; Maciá-Soler }{ }^{(19)}\end{array}$ & $\begin{array}{l}\text { Nursing professionals and health care assistants' } \\
\text { perception of patient safety culture in the operating } \\
\text { room] }\end{array}$ & 2015 \\
\hline & C3 & $\begin{array}{l}\text { Nugent; Hseino; Ryan; } \\
\text { Neary; Keane } \\
\end{array}$ & $\begin{array}{l}\text { The surgical safety checklist survey: A national } \\
\text { perspective on patient safety }\end{array}$ & 2013 \\
\hline & $\mathrm{C} 4$ & Donmez; Ozbayir ${ }^{(21)}$ & $\begin{array}{l}\text { Validity and reliability of the 'good perioperative } \\
\text { nursing care scale' for Turkish patients and nurses }\end{array}$ & 2011 \\
\hline
\end{tabular}


Apesar da contribuição de diversos autores, o modelo de avaliação da qualidade de prestação de cuidados de saúde, usado actualmente, é o modelo de Donabedian, proposto pela OMS ${ }^{(5)}$. Donabedian, considerado o grande mestre da qualidade em saúde ${ }^{(22)}$, desenvolveu um modelo de avaliação da qualidade que se tornou o padrão no domínio dos serviços de saúde ${ }^{(23)}$. Este modelo foi introduzido pela primeira vez em $1966^{(24)}$ e consiste na avaliação da qualidade em saúde, assente em três componentes essenciais: a estrutura, o processo e os resultados ${ }^{(22)}$.

\section{Avaliação da Estrutura}

A estrutura refere-se às características mais estáveis e serve para designar as condições em que os cuidados são prestados. Os atributos da estrutura são mais facilmente observáveis e mais facilmente documentados e tendem por sua vez a ser mais estáveis ${ }^{(24)}$.

$\mathrm{Na}$ análise global dos artigos analisados são descritos poucos indicadores referentes à estrutura ${ }^{(9,10)}$. No estudo A1, os autores consideram que a qualidade pode ser controlada através de uma equipa fixa em casos consecutivos semelhantes ${ }^{(9)}$. Numa era marcada pela eficiência e avaliação do custobenefício dos cuidados de saúde, onde os serviços cirúrgicos são dispendiosos, é importante para as instituições de saúde usá-los de modo eficiente. Através de um estudo de caso-controle A1, o autor salienta que existe impacto com a utilização de casos consecutivos semelhantes, nomeadamente no tempo de procedimento, assim como no custo das cirurgias através de uma melhor programação de recursos cirúrgicos. Estes resultados são bem conhecidos para os processos de fabrico industrial ${ }^{(9)}$.

No estudo A2, sobre a utilização do Seis Sigma e técnicas de estatística para controlo e melhoria da qualidade no bloco operatório, refere que temos sido lentos na incorporação de técnicas de confiabilidade industrial no bloco operatório(10). O Seis Sigma é uma abordagem de melhoria de qualidade que tem por objectivo reduzir o número de defeitos. Nas últimas três décadas, ajudou várias empresas a aumentar a capacidade de seus processos e aumentar o nível de qualidade do seu produto ou serviço ${ }^{(25)}$.

Ao longo do estudo A2, os tempos de espera entre as cirurgias foram avaliados pela auditoria dos registos de sala de operação de um hospital durante um período de 1 mês ${ }^{(10)}$. Os registos de 628 doentes submetidos à cirurgia de cólon ao longo de um período de 5 anos foram analisados. O tempo médio de espera entre os casos foi de 53 minutos. O que revela que, no geral, esta instituição tem 331 horas de tempo não produtivo entre procedimentos programados. Os autores sugerem algumas medidas a ser incluídas: melhoria na preparação pré-operatória do doente, maior eficiência do tempo de espera pelo cirurgião entre as cirurgias, e que o tradicional agendamento e bloqueio dos tempos cirúrgicos sejam substituídos por uma abordagem de base industrial "Just in time"(10).

\section{Avaliação do Processo}

O processo refere-se a todas as actividades que constituem os cuidados de saúde. Os indicadores de processo permitem avaliar a forma como um protocolo de cuidados de saúde é realizado ou prestado $^{(5)}$. Dos artigos analisados são realçados instrumentos relevantes da área dos processos da avaliação da qualidade ${ }^{(11-17)}$.

No estudo B4, é salientada a importância do trabalho em equipa para minimizar os erros e efeitos adversos no bloco operatório, ao longo do qual traduzem e validam a escala "Observational Teamwork Assessment for Surgery" (OTAS) ${ }^{(14)}$. A Escala incorpora cinco dimensões: comunicação, coordenação, cooperação, liderança e monitorização. Cada comportamento é pontuado numa escala de 7 pontos, variando de 0 a 6 , em que 0 indica o comportamento problemático que dificulta severamente a equipa e 6 indica o trabalho em equipa exemplar que é altamente eficaz em melhorar o funcionamento da equipa. Este instrumento pode ser utilizado para potenciar o trabalho em equipa e o desenvolvimento de competências interpessoais para os profissionais que trabalham em blocos operatórios ${ }^{(14)}$. 
No trabalho em equipa é preponderante a comunicação, evidenciada no estudo B2, como forma de minimizar o erro no bloco operatório ${ }^{(12)}$. Os autores realizaram um estudo de intervenção com grupo de controlo, através da implementação da ferramenta de comunicação "Situation, Background, Assessment, Recommendations (SBAR)". Esta ferramenta é utilizada nas organizações de alto risco para tornar a comunicação mais eficaz e consistente, tendo sido introduzida na área da saúde. Para avaliação do impacto da intervenção, os autores utilizaram antes e após a intervenção duas escalas ("NursePhysician Questionnaire","Spreitzer's empowerment scale"), concluindo que a implementação do SBAR pode melhorar a comunicação entre os profissionais, melhorar o clima de segurança e reduzir incidentes causados por erros de comunicação ${ }^{(12)}$.

Segundo o estudo B7, as distracções no bloco operatório contribuem para a ocorrência de eventos adversos, sobre as quais os autores realizaram um estudo observacional. Foram analisadas 32 cirurgias, ao longo das quais foram observados 3557 eventos potencialmente perturbadores, dos quais 1173 (33\%) foram considerados por distracção ${ }^{(17)}$.

A Aliança Mundial para a Segurança do Doente, da Organização Mundial de Saúde (OMS), identificou dez objectivos essenciais para a segurança cirúrgica, esses objectivos estão compilados na lista de verificação cirúrgica ou "checklist", cujo objetivo é reforçar as práticas de segurança e promover uma melhor comunicação e trabalho de equipa multidisciplinar ${ }^{(5)}$. Alguns destes aspectos já foram referidos nos estudos apresentados, nomeadamente a comunicação, o trabalho em equipa e as distrações, sendo agora realçado os instrumentos para garantir a segurança, nomeadamente a checklist, o "Briefing and debriefing" e os cinco passos para a cirurgia segura.

O conceito de checklist divulgado e emanado pela OMS como linha central de orientação tem sido largamente utilizado ${ }^{(5)}$. No entanto, no estudo B5, realizado em hospitais ingleses, a conformidade com a Checklist de Segurança Cirúrgica da OMS é muito inferior à indicada pelos dados administrativos, revelando que a observação directa do desempenho real possa estar abaixo do ideal ${ }^{(15)}$.

No estudo B3, que aborda a implementação da checklist, os autores concluíram que esta melhorou o trabalho em equipa e o clima de segurança em um hospital universitário no Japão. As mudanças no trabalho em equipe e o clima de segurança após aplicação da checklist foram validados através da escala "Safety Attitudes Questionnaire (SAQ)". O SAQ é uma escala, com 12 itens que inclui a pesquisa de fatores humanos e tem sido extensivamente validada em diferentes serviços de saúde ${ }^{(13)}$.

Os conceitos "briefing e debriefing" são aplicados no estudo $\mathrm{B} 6^{(16)}$. Estes instrumentos têm sido utilizados entre os pilotos comerciais e de combate, e foram formalizadas para formação pela National Aeronautics and Space Administration (NASA), e agora aplicados à área da saúde, primeiramente nos blocos operatórios. O Briefing estabelece uma plataforma de entendimento comum e planeamento colaborativo. E o debriefing constitui um relatório em tempo real e pode identificar "quase-acidentes", que geralmente não são detectados pelo registo. Ao longo deste momento, são reconstruidos todos os eventos com ênfase na segurança, comunicação, eficiência e potencial de melhoria. Estes instrumentos evidenciaram ter impacto na atitude da equipa e na segurança do doente ${ }^{(16)}$.

Por último, "Os cinco passos para uma cirurgia segura" foram implementados no estudo B1 (11). "Os cinco passos para uma cirurgia segura" (5 Steps to Safer Surgery (5SSS)) incorporam um "briefing", os três passos da lista de verificação da OMS Surgical Safety (SSC) e um"debriefing". Para avaliação desta intervenção foi aplicado o Safety Attitude Questionnaire - Operating Room (SAQ-OR), tendo obtido impacto nas dimensões: clima de segurança, percepção da gestão, condições de trabalho, satisfação no trabalho e trabalho em equipa ${ }^{(11)}$.

\section{Avaliação do Resultado}

Os indicadores de resultado permitem avaliar os resultados ou impacto sobre a saúde da população(5). O resultado reflecte as mudanças, no sentido desejável ou indesejável, que são provocadas no estado de saúde do indivíduo ou populações que podem ser atribuídas aos cuidados de saúde. Também podem ser considerados como resultados as mudanças relacionadas com conhecimentos e comportamentos, bem como a satisfação do cliente/família e profissionais. Alguns destes indicadores são apresentados nos artigos analisados ${ }^{(18-21)}$. 
Um dos resultados indesejáveis das cirurgias são as complicações pós-operatórias, decorrente deste indicador. Os autores do estudo C1 propõem a utilização de um instrumento "IRT30" usado para monitorizar as complicações cirúrgicas de forma objetiva, analisando todos os retornos à sala de operações até 30 dias após a cirurgia primária(18).

Os autores do estudo C2 avaliam as percepções e opiniões dos enfermeiros de um bloco operatório sobre a segurança do doente ${ }^{(19)}$. Através de um estudo transversal, observacional, realizado utilizando a versão espanhola do questionário "Hospital Survey on Patient Safety Culture", concluem que as principais áreas que necessitam de intervenção são a melhor dotação dos serviços e apoio da gestão em matéria de segurança do doente. O questionário "Hospital Survey on Patient Safety Culture" é composto por 42 itens relacionados com as percepções dos profissionais sobre a cultura da segurança do paciente em 12 dimensões: duas dimensões de resultado, sete dimensões sobre a unidade e três sobre o hospital ${ }^{(19)}$.

Por outro lado, no estudo C4 é avaliado o grau de satisfação dos doentes sobre a qualidade de assistência de enfermagem perioperatória, através do instrumento "Good Perioperative Nursing Care Scale" (GPNCS) ${ }^{(21)}$. A escala é uma escala do tipo Likert (0-5), contém 34 itens, com seis dimensões: cuidados físicos, fornecer informações, apoio, respeito, características do pessoal, ambiente e processo de enfermagem ${ }^{(21)}$.

Por último, os autores do estudo C3 avaliam nos hospitais da Irlanda, a percepção dos profissionais do bloco operatório sobre a utilização da "checklist", através de um questionário on-line. Estes autores concluem que a "checklist" não foi implementada em todos os blocos operatórios na Irlanda ${ }^{(20)}$. Nos locais em que foi aplicado percepciona-se uma mudança positiva de cultura de segurança. No entanto, de modo a optimizar os benefícios potenciais associados a este instrumento de segurança, será necessário maior formação, apoio, trabalho em equipa e comunicação. Sobre a qualidade e segurança no atendimento cirúrgico, os autores salientam que a mesma requer mais do que apenas uma lista de verificação, exige trabalho em equipa, incluindo muitos outros ingredientes, nomeadamente a abertura de canais de comunicação, recursos, feedback e auditoria ${ }^{(20)}$.

\section{CONCLUSÃO}

A qualidade no bloco operatório tem sido amplamente divulgada nos últimos anos, embora mais direccionada ao risco e à segurança do doente, associadas às orientações emanadas pela OMS. O presente estudo pretendeu agrupar o conhecimento existente sobre os instrumentos para avaliar a qualidade no bloco operatório através de uma revisão integrativa, estimulando a discussão em torno da importância da avaliação da qualidade no bloco operatório.

Da revisão integrativa realizada dos estudos em análise, foram identificadas três áreas temáticas, sobreponíveis ao modelo de Donabedian, e proposto pela OMS, nomeadamente a Estrutura, o Processo e o Resultado.

Os achados deste estudo revelam instrumentos mais frequentes no âmbito do processo, e essencialmente associados à cultura de segurança e risco proposto pelas diretivas da OMS, realçase também a necessidade de maior desenvolvimento de instrumentos válidos e mais abrangentes, integrando as diferentes componentes da avaliação da qualidade, a estrutura, o processo e o resultado. Realçando que a força do modelo de Donabedian reside nas relações entre estes indicadores, ou seja, a estrutura influencia o processo e o processo por sua vez, influencia o resultado. Pelo que a avaliação global dos cuidados de saúde prestados exige a compreensão dos três elementos, individualmente, mas também as relações entre eles.

\section{O REFERÊNCIAS}

1. Instituto Nacional de Estatística - INE [Internet]. Portugal: Portal da estatística da saúde. Conceitos por tema. 2015 [acesso em 06 jan 2015]. Disponível: http://smi.ine.pt/Conceito/Detalhes/1676

2. Ministério da Saúde (PT). Avaliação da situação nacional dos blocos operatórios. [Internet] 2015 [acesso em 
06 jan 2016]. Disponível: http://www2.portaldasaude.pt/portal/conteudos/a+saude+em+portugal/publicacoes/ estudos/relatorio+bo.htm\#sthash.v9jzzyJC.dpuf

3. Caspe Healthcare Knowledge System (CKHS). Programa de acreditação internacional para organizações de saúde, normas para a acreditação. 3. ${ }^{a}$ Edição, Versão 01; 2010.

4. Fragata J. Erros e acidentes no bloco operatório: revisão do estado da arte. Rev Port Saúde Pública. [Internet] 2010; Vol Temat(10) [acesso em 06 jan 2016]. Disponível: http://www.elsevier.pt/pt/revistas/revista-portuguesasaude-publica-323/artigo/erros-e-acidentes-no-bloco-operatorio-revisao-do-13189855

5. Direcção-Geral de Saúde (PT). Orientações da OMS para a Cirurgia Segura 2009: cirurgia segura salva vidas. World Health Organization. [Internet] 2010 [acesso em 06 jan 2016]. Disponível:

http://www.dgs.pt/ms/8/paginaRegisto.aspx?back=1\&id=16317

6. Styer KA, Ashley SW, Schmidt I, Zive EM, Eappen S. Implementing the world health organization surgical safety checklist: a model for future perioperative initiatives. AORN Journal. [Internet] 2011; 94(6) [acesso em 06 jan 2016]. Disponível: https://dx.doi.org/10.1016/j.aorn.2011.03.012

7. Valderrama-Zurián JC, Aguilar-Moya R, Melero-Fuentes D, Aleixandre-Benavent R. A systematic analysis of duplicate records in Scopus. Journal of Informetrics. [Internet] 2015; 9(3) [acesso em 06 jan 2016]. Disponível: https://dx.doi.org/10.1016/j.joi.2015.05.002

8. de Souza M, da Silva M, de Carvalho R. Revisão integrativa: o que é e como fazer?. Einstein V. [Internet] 2010; 8(1 Pt 1) [acesso em 06 jan 2016]. Disponível:

http://apps.einstein.br/revista/arquivos/PDF/1134-Einsteinv8n1_p102-106_port.pdf

9. Stepaniak PS, Vrijland WW, De Quelerij M, De Vries G, Heij C. Working with a fixed operating room team on consecutive similar cases and the effect on case duration and turnover time. Arch Surg. [Internet] 2010; 145(12) [acesso em 06 jan 2016]. Disponível: https://dx.doi.org/10.1001/archsurg.2010.255

10. Sedlack JD. The utilization of six sigma and statistical process control techniques in surgical quality improvement. J Healthc Qual. [Internet] 2010; 32(6) [acesso em 06 jan 2016]. Disponível:

https://dx.doi.org/10.1111/j.1945-1474.2010.00102.x

11. Hill MR, Roberts MJ, Alderson ML, Gale TCE. Safety culture and the 5 steps to safer surgery: An intervention study. Br J Anaesth. [Internet] 2015; 114(6) [acesso em 06 jan 2016]. Disponível:

https://dx.doi.org/10.1093/bja/aev063

12. Randmaa M, Mårtensson G, Swenne CL, Engström M. SBAR improves communication and safety climate and decreases incident reports due to communication errors in an anaesthetic clinic: A prospective intervention study. BMJ Open. [Internet] 2014; 4(1) [acesso em 06 jan 2016]. Disponível:

https://dx.doi.org/10.1136/bmjopen-2013-004268

13. Kawano T, Taniwaki M, Ogata K, Sakamoto M, Yokoyama M. Improvement of teamwork and safety climate following implementation of the WHO surgical safety checklist at a university hospital in Japan. J Anesth. [Internet] 2014; 28(3). [acesso em 06 jan 2016] Disponível: https://dx.doi.org/10.1007/s00540-013-1737-y

14. Arias AAC, Barajas R, Eslava-Schmalbach JH, Wheelock A, Duarte GH, Hull L, Sevdalis N. Translation, cultural adaptation and content re-validation of the observational teamwork assessment for surgery tool. Int J Surg. [Internet] 2014; 12(12) [acesso em 06 jan 2016]. Disponível: https://dx.doi.org/10.1016/j.ijsu.2014.10.001

15. Pickering SP, Robertson ER, Griffin D, Hadi M, Morgan LJ, Catchpole KC, McCulloch P. Compliance and use of the world health organization checklist in UK operating theatres. British Journal of Surgery. [Internet] 2013; 100(12) [acesso em 06 jan 2016]. Disponível: https://dx.doi.org/10.1002/bjs.9305

16. Papaspyros SC, Javangula KC, Adluri RKP, O'Regan DJ. Briefing and debriefing in the cardiac operating room. Analysis of impact on theatre team attitude and patient safety. Interact Cardiovasc Thorac Surg. [Internet] 2010; 10(1) [acesso em 06 jan 2016]. Disponível: https://dx.doi.org/10.1510/icvts.2009.217356

17. Jothiraj H, Howland-Harris J, Evley R, Moppett IK. Distractions and the anaesthetist: A qualitative study of context and direction of distraction. Br J Anaesth. [Internet] 2013; 111(3). [acesso em 06 jan 2016]. Disponível: https://dx.doi.org/10.1093/bja/aet108 
18. Sukha A, Li E, Sykes T, Fox A, Schofield A, Houghton A. Inadvertent returns to theatre within 30 days (IRT30) of surgery: An educational tool to monitor surgical complications and improve our performance as surgeons. Clinical Governance. [Internet] 2015; 20(4) [acesso em 06 jan 2016]. Disponível:

https://dx.doi.org/10.1108/CGIJ-03-2015-0011

19. Bernalte-Martí V, Orts-Cortés MI, Maciá-Soler L. Nursing professionals and health care assistants' perception of patient safety culture in the operating room. Enferm Clin. [Internet] 2015; 25(2) [acesso em 06 jan 2016]. Disponível: https://dx.doi.org/10.1016/j.enfcli.2014.08.002

20. Nugent E, Hseino H, Ryan K, Traynor O, Neary P, Keane FBV. The surgical safety checklist survey: A national perspective on patient safety. Ir J Med Sci. [Internet] 2013; 182(2) [acesso em 06 jan 2016]. Disponível:

https://dx.doi.org/10.1007/s11845-012-0851-4

21. Donmez YC, Ozbayir T. Validity and reliability of the 'good perioperative nursing care scale' for Turkish patients and nurses. J Clin Nurs. [Internet] 2011; 20(1-2) [acesso em 06 jan 2016]. Disponível:

https://dx.doi.org/10.1111/j.1365-2702.2010.03314.x

22. Mezomo JC. Gestão da qualidade na saúde: princípios básicos. $1^{\text {a }}$ ed. São Paulo, Brasil: Editora Manole; 2001.

23. Revere L, Black K, Huq A. Integrating Six Sigma and CQI for improving patient care. The TQM Magazine. [Internet] 2004; 16(2) [acesso em 06 jan 2016]. Disponível: https://dx.doi.org/10.1108/09544780410522991

24. Donabedian A. An introduction to quality assurance in health care. New York: Oxford University Press; 2003.

25. Hassan R, Marimuthu M, Mahinderjit-Singh M. Application of six-sigma for process improvement in manufacturing industries: A case study. International Business Management. [Internet] 2016; 10(5) [acesso em 06 jan 2016]. Disponível: https://dx.doi.org/10.3923/ibm.2016.676.691 